\title{
Large dust particles in disks around T Tauri stars
}

\author{
J. Rodmann ${ }^{1}$, Th. Henning ${ }^{1}$, C. J. Chandler ${ }^{2}$, L. G. Mundy ${ }^{3}$, and D. J. Wilner ${ }^{4}$ \\ 1 Max-Planck-Institut für Astronomie, Königstuhl 17, 69117, Heidelberg, Germany \\ e-mail: rodmann@mpia.de \\ 2 National Radio Astronomy Observatory, PO Box O, Socorro, NM 87801, USA \\ 3 Department of Astronomy, University of Maryland, College Park, MD 20742, USA \\ ${ }^{4}$ Harvard-Smithsonian Center for Astrophysics, 60 Garden Street, Cambridge, MA 02138, USA
}

Received 12 August 2005 / Accepted 14 September 2005

\begin{abstract}
We present 7-mm continuum observations of 14 low-mass pre-main-sequence stars in the Taurus-Auriga star-forming region obtained with the Very Large Array with $\sim 1$ '. 5 resolution and $\sim 0.3$ mJy rms sensitivity. For 10 objects, the circumstellar emission has been spatially resolved. The large outer disk radii derived suggest that the emission at this wavelength is mostly optically thin. The millimetre spectral energy distributions are characterised by spectral indices $\alpha_{\mathrm{mm}}=2.3$ to 3.2. After accounting for contributions from free-free emission and corrections for optical depth, we determine dust opacity indices $\beta$ in the range 0.5 to 1.6 , which suggest that millimetre-sized dust aggregates are present in the circumstellar disks. Four of the sources with $\beta>1$ may be consistent with submicron-sized dust as found in the interstellar medium. Our findings indicate that dust grain growth to millimetre-sized particles is completed within less than 1 Myr for the majority of circumstellar disks.
\end{abstract}

Key words. stars: pre-main sequence - stars: planetary systems: protoplanetary disks - planetary systems: formation

\section{Introduction}

Although dust grains constitute only a minor fraction of the disk material around young stars $(\sim 1 \mathrm{Myr})$, they play a pivotal role in the complicated multi-stage process of planet formation. The growth of submicron-sized dust particles as found in the interstellar medium (ISM) to larger aggregates by coagulation (collisional sticking) is the first essential step towards building larger rocky bodies (planetesimals) that may eventually accrete into terrestrial planets and planetary cores (Beckwith et al. 2000).

Statistical analysis of the near-infrared excess of young stellar clusters shows that disk emission disappears within a few Myr (Strom et al. 1989; Haisch et al. 2001). Similarly, sub-mm and $\mathrm{mm}$ observations suggest a decrease in the amount of cold circumstellar dust during the post T-Tauri phase (Carpenter et al. 2005). The decline of dust emission can be interpreted as a consequence of gradual mass loss through disk dissipation and/or opacity changes due to particle growth. It is important to stress that such studies only trace the evolution of the dust disk. The evolution of the gaseous disk, containing the bulk of the disk mass, is at present only poorly understood.

For many years there has been little sound observational evidence for dust grain growth in disks around pre-mainsequence stars. The analysis of spectral energy distributions showed that the sub- $\mathrm{mm} / \mathrm{mm}$ fluxes of $\mathrm{T}$ Tauri stars decline more slowly towards longer wavelengths than expected for ISM-sized dust (e.g. Beckwith \& Sargent 1991). It was tempting to interpret a shallow spectral slope as an indication of the presence of particles much larger than in the ISM $(\gg 0.1 \mu \mathrm{m})$. It was soon realised, however, that spatially unresolved disk observations cannot be used to distinguish between small, optically thick disks containing sub-micron ISM dust and extended, optically thin disks with larger dust particles. Spatially resolved images are needed to break this parameter degeneracy (Koerner et al. 1995; Dutrey et al. 1996).

Recent technical improvements at the Very Large Array (VLA) allowed the resolution of the disk around the young star TW Hya at $7 \mathrm{~mm}$ and the determination of the dust opacity index (Wilner et al. 2000; Calvet et al. 2002). Testi et al. (2003) resolved the dusty disk around the pre-main-sequence star CQ Tau and concluded that millimetre- and even centimetresized dust particles must be present in the outer disk. In a similar study of six isolated intermediate-mass (Herbig Ae) stars by Natta et al. (2004), dust grain growth was inferred for two objects.

In this paper, we report the results of 7-mm continuum observations of $14 \mathrm{~T}$ Tauri stars located in the Taurus-Auriga starforming region, currently the largest sample of low-mass premain-sequence stars investigated for signs of dust grain growth to millimetre/centimetre particle sizes. 
Table 1. Properties of sample stars.

\begin{tabular}{|c|c|c|c|c|c|c|c|c|}
\hline \multirow[t]{2}{*}{ Star } & \multicolumn{2}{|c|}{ Coordinates (J2000.0) } & \multirow{2}{*}{$\begin{array}{c}\text { Spectral } \\
\text { type }^{a}\end{array}$} & \multirow{2}{*}{$\begin{array}{l}T_{\mathrm{eff}}^{b} \\
(\mathrm{~K})\end{array}$} & \multirow{2}{*}{$\begin{array}{l}M_{\star}^{c} \\
\left(M_{\odot}\right)\end{array}$} & \multirow{2}{*}{$\begin{array}{c}L_{\star}^{b} \\
\left(L_{\odot}\right)\end{array}$} & \multirow{2}{*}{$\begin{array}{l}\text { Age }^{c} \\
(\mathrm{Myr})\end{array}$} & \multirow[t]{2}{*}{ Location } \\
\hline & $\alpha(\mathrm{h} \mathrm{m} \mathrm{s})$ & $\delta\left({ }^{\circ}, \prime \prime\right)$ & & & & & & \\
\hline CY Tau & 41733.74 & +282046.5 & M1 & 3720 & 0.48 & 0.47 & 0.7 & L 1495 \\
\hline RY Tau & 42157.42 & +282635.5 & $\mathrm{~K} 1$ & 5080 & 1.69 & 7.60 & 0.2 & L 1495 \\
\hline FT Tau & 42339.19 & +245614.2 & cont. & $3890^{c}$ & - & - & - & В 217 \\
\hline DG Tau B & 42702.56 & +260530.4 & - & - & - & - & - & B 217 \\
\hline DG Tau & 42704.69 & +260616.1 & M & $3890^{c}$ & 0.56 & $1.7^{c}$ & 0.3 & B 217 \\
\hline HL Tau & 43138.41 & +181357.6 & K7-M2 & 4060 & 0.55 & $0.9^{c}$ & 1.0 & L 1551 \\
\hline GG Tau & 43230.39 & +173140.1 & K7 & 4060 & 0.65 & 1.50 & 0.3 & B 217 \\
\hline UZ Tau E & 43243.10 & +255231.0 & M1-3 & 3680 & 0.44 & $2.94^{c}$ & $<0.1$ & B 217 \\
\hline DL Tau & 43339.09 & +252037.9 & K7 & 4060 & 0.56 & $0.77^{c}$ & 1.2 & TMC 2 \\
\hline DM Tau & 43348.74 & +181010.0 & M1 & 3720 & 0.62 & 0.25 & 3.2 & L 1551 \\
\hline CI Tau & 43352.03 & +225030.2 & K7 & 4060 & 0.70 & 0.87 & 0.8 & L 1536 \\
\hline DO Tau & 43828.59 & +261049.4 & M0 & 3850 & 0.72 & 1.20 & 0.6 & TMC 1 \\
\hline $\mathrm{LkCa} 15$ & 43917.81 & +222103.6 & K5 & 4350 & - & 0.74 & $1.0^{e}$ & L 1536 \\
\hline GM Aur & 45510.98 & +302159.5 & $\mathrm{~K} 3$ & 4730 & 0.72 & 0.83 & 1.8 & L 1517 \\
\hline
\end{tabular}

References: ${ }^{a}$ Herbig \& Bell (1988), ${ }^{b}$ Kenyon \& Hartmann (1995), ${ }^{c}$ Beckwith et al. (1990), ${ }^{d}$ Leinert et al. (1993), ${ }^{e}$ Duvert et al. (2000).

\section{Observations}

\subsection{Sample of objects}

Our sample consists of 14 low-mass pre-main-sequence stars located towards the Taurus-Auriga star formation region (Table 1). All sample stars are catalogued as classical T Tauri stars, based on the equivalent width of their $\mathrm{H} \alpha$ emission line $\gtrsim 10 \AA$ (Herbig \& Bell 1988). They are classified as Class II young stellar objects, as derived from the slope of their infrared spectral energy distributions, $\alpha_{\mathrm{IR}}=\operatorname{dlog}\left(\lambda F_{\lambda}\right) / \operatorname{dlog} \lambda$, with $-2 \lesssim \alpha \lesssim 0$ (Adams et al. 1987; Kenyon \& Hartmann 1995; Hartmann 2002).

Observations of the millimetre continuum emission have confirmed the presence of circumstellar disks around these $\mathrm{T}$ Tauri stars, with disk masses ranging from 0.02 to $0.7 M_{\odot}$ (Beckwith et al. 1990; Dutrey et al. 1996). For the majority of the stars in our sample, emission from circumstellar CO gas has been detected, often tracing a disk in Keplerian rotation (Koerner et al. 1993; Dutrey et al. 1994; Koerner \& Sargent 1995; Handa et al. 1995; Dutrey et al. 1996; Jensen et al. 1996; Mitchell et al. 1997; Duvert et al. 2000; Najita et al. 2003).

The stellar ages and masses have been determined by fitting pre-main-sequence isochrones to loci of the stars in the Hertzsprung-Russell diagram; they range from 0.1 to $3.2 \mathrm{Myr}$ and from 0.4 to $1.7 M_{\odot}$, respectively (Beckwith et al. 1990; Duvert et al. 2000). The uncertainties of the age determinations are considerable and preclude a meaningful correlation with the opacity indices $\beta$.

The average distance to the Taurus-Auriga molecular cloud as found in the literature varies between $135 \mathrm{pc}$ (Cernicharo et al. 1985) and $160 \mathrm{pc}$ (Strom et al. 1989). We adopt the standard value of 140 pc (Elias 1978; Kenyon et al. 1994; Loinard et al. 2005) to convert angular scales into physical sizes.

\subsection{7-mm continuum observations}

The NRAO ${ }^{1}$ Very Large Array was used to observe the dust continuum emission of $14 \mathrm{~T}$ Tauri stars at $43.34 \mathrm{GHz}(7 \mathrm{~mm})$. The total receiver bandwidth was $100 \mathrm{MHz}$, divided into two $50 \mathrm{MHz}$ bands centred at $43.3149 \mathrm{GHz}$ and $43.3649 \mathrm{GHz}$. The observations were carried out in $\mathrm{D}$ configuration on 22-23 March 2003 and again on 12 May 2003. The 27 VLA antennas provided $27 \times(27-1) / 2=351$ baselines from $35 \mathrm{~m}$ to $1.03 \mathrm{~km}$, corresponding to $5-150 \mathrm{k} \lambda$ at $7 \mathrm{~mm}$. The May observations took place during array reconfiguration (D to $A$ ); only 26 antennas were available.

Phase calibration was accomplished by sandwiching the on-source observations between pointings of nearby secondary calibrators (radio sources J0431+2037, J0403+2600, $\mathrm{J} 0443+3441)$. The typical source/phase calibrator cycle time was 5-10 min. Absolute flux calibration was obtained from observation of the quasar J0542+4951 (3C 147) which is assumed to have a flux density of $0.91 \mathrm{Jy}$. The estimated uncertainty in the absolute flux calibration is $\sim 10 \%$. Reference pointing measurements were made approximately every hour.

The $(u, v)$ data sets for the three observing dates were merged using the $\mathcal{A} \mathcal{I P S}$ task DBCON. CLEANed images were obtained with IMAGR using a ROBUST parameter of 0 , which is intermediate between natural and uniform weighting of the visibility data and optimises for spatial resolution and sensitivity. Three weak sources (DM Tau, CI Tau, and LkCa 15) were CLEANed using natural weighting (ROBUST=5) to increase signal-to-noise ratio and allow secure detection. The extended emission of GG Tau is also best imaged using natural weighting. Figure 1 shows the corresponding contour plots for the 7 -mm images.

\footnotetext{
1 The National Radio Astronomy Observatory is operated for the National Science Foundation by Associated Universities, Inc., under a cooperative agreement.
} 

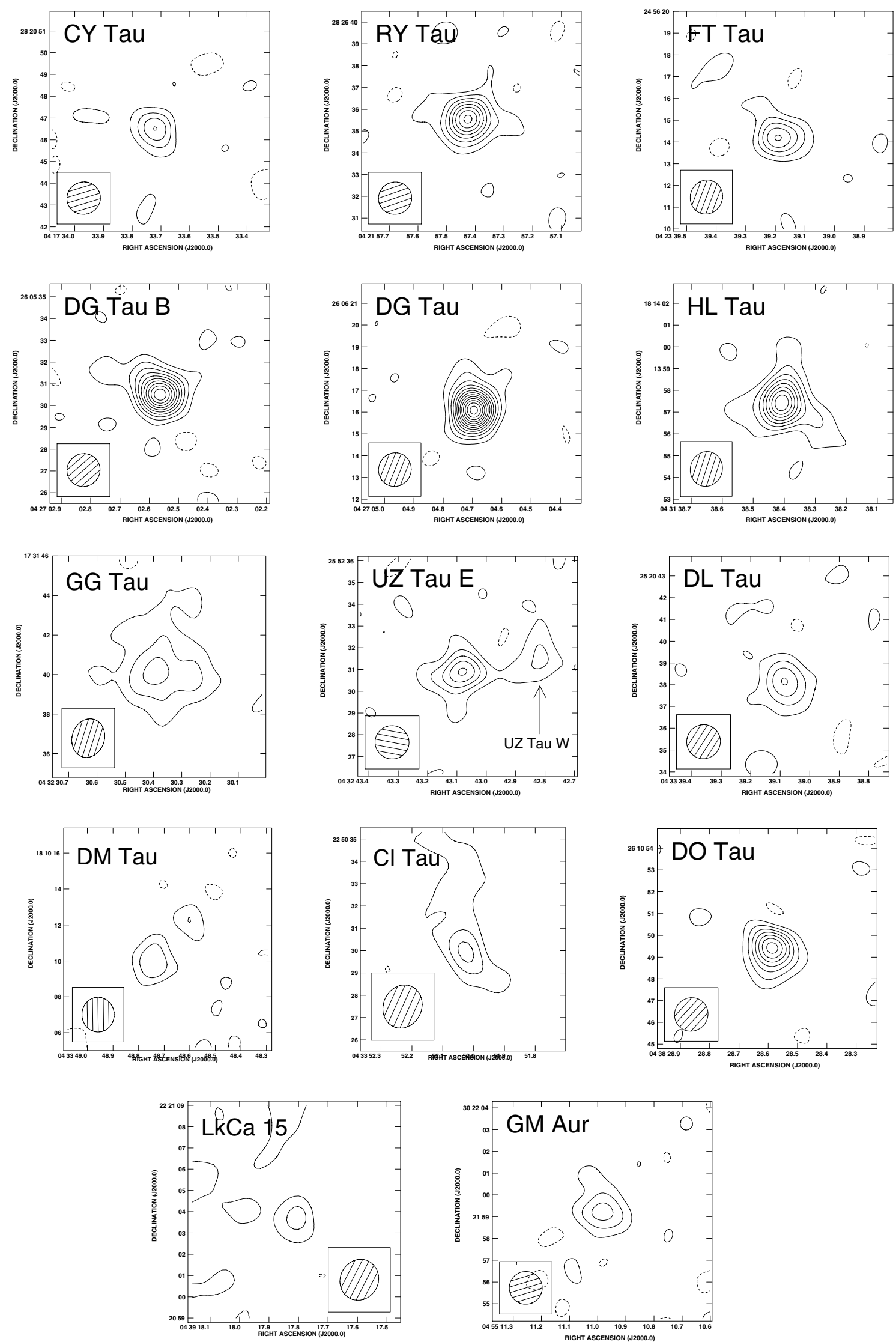

Fig. 1. VLA D-configuration images of the $\lambda=7 \mathrm{~mm}$ continuum emission. Contour levels are drawn in steps of the corresponding $2 \sigma$ rms noise level; the average $1 \sigma \mathrm{rms}$ noise level is $\sim 0.15 \mathrm{mJy} / \mathrm{beam}$. The synthesized beam is shown in the bottom left corner of each panel; the average beam size is $\sim 1^{\prime \prime} .5$. 


\subsection{Centimetre observations}

Five of the sources have poor-quality archival VLA detections at $8 \mathrm{GHz}$ (Wilner 1998). All five sources were reobserved at $3.6 \mathrm{~cm}(8.46 \mathrm{GHz}), 2.0 \mathrm{~cm}(14.94 \mathrm{GHz})$, and $1.3 \mathrm{~cm}$ $(22.46 \mathrm{GHz})$. The total bandwidth was again $100 \mathrm{MHz}$, with two $50 \mathrm{MHz}$ channels $50 \mathrm{MHz}$ apart. The $1.3-\mathrm{cm}$ observations were carried out on 17 March 2003; the 2.0- and 3.6-cm observations on 21 March 2003. All observations were made in the compact D configuration, covering baselines of $3-77 \mathrm{k} \lambda$, $2-50 \mathrm{k} \lambda$, and $1-30 \mathrm{k} \lambda$ in the $(u, v)$-plane at $1.3,2.0$, and $3.6 \mathrm{~cm}$, respectively.

The flux density scale was set by observations of the calibrator J0542+4951 with known flux densities of 1.80, 2.71, and $4.74 \mathrm{Jy}$ at $1.3,2.0$, and $3.6 \mathrm{~cm}$, respectively. CLEANed images were produced with IMAGR and natural weighting of the visibilities (ROBUST=5). Finally, primary beam corrections were performed using the $\mathcal{A I P S}$ task LTESS to compensate for the non-uniform single-antenna response across the field-of-view of the entire array. The contour maps are shown in Fig. 2; note that DG Tau and DG Tau B appear in the same image.

\subsection{Radio seeing}

Short-term variations in precipitable water vapour lead to changes in the path length of an electromagnetic wave travelling through the troposphere, causing phase fluctuations when observed by radio interferometers. Tropospheric phase noise negatively affects spatial resolution and coherence, especially at mm wavelengths (Carilli et al. 1999).

In order to estimate the amount of smearing introduced by tropospheric phase noise, we compared self-calibrated and standard-calibrated images of two bright radio sources. Images of self-calibrated sources are corrected for the radio seeing on short timescales (3.3 s integration time), and deconvolution will give the intrinsic source size. The same procedure applied to images obtained from ordinary calibration gives the "seeing disk". By using two test sources, one unresolved $(\mathrm{J} 0426+2327)$, the other extended $(\mathrm{J} 0412+2305)$, we checked the flux reduction by phase-noise induced smearing for point sources and extended sources, respectively.

The analysis of the merged 7-mm data showed that the overall radio seeing was $\sim 0$.' 5 . Inspection of the single-day data revealed that the seeing on the second day (23 March 2003) was the worst $(\sim 0.7)$, while on 22 March and 12 May better water vapour conditions prevailed (about $0 . ' 1$ and 0.4 , respectively). The integrated 7-mm fluxes are reduced by 4-5\%, and are within $2 \%$ for each day.

The seeing at $1.3 \mathrm{~cm}$ was about $0 .{ }^{\prime} 5$. The peak fluxes might be reduced by up to $5 \%$; the integrated fluxes were affected less than $1 \%$. Tropospheric seeing effects at longer centimetre wavelengths are expected to be negligible.

\section{Results}

\subsection{7-mm fluxes and disk sizes}

We detected the continuum emission from cold circumstellar dust at $7 \mathrm{~mm}$ for all $14 \mathrm{~T}$ Tauri stars comprising our sample.
Two sources (DM Tau and LkCa 15) have signal-to-noise ratios $<5$, and we do not consider these sources further in our analysis. The integrated fluxes were measured on the combined maps using the $\mathcal{A I P S}$ routine IMSTAT, and corrected for seeing reduction. In the light of the radio seeing analysis, we decided to use the excellent conditions of the first-day data set to determine source sizes. The task IMFIT was used to check if the source was spatially resolved or unresolved by fitting a two-dimensional Gaussian function to the emission peak. The deconvolved disk sizes are of the order 100-200 AU, representing typical dimensions for protoplanetary disks. The major and minor axes can, in principle, be used to derive the inclination angle of the disks. Their uncertainties, however, are very large, and the disk orientations are poorly constrained. Table 2 summarises the results derived from the 7-mm maps.

The extended dust emission of three sources is of particular interest. The map of HL Tau shows a prominent "finger" pointing in south-western direction. The measured position angle of $235^{\circ} \pm 10^{\circ}$ is roughly co-aligned with the counter-jet of HL Tau (Mundt et al. 1988; Wilner \& Lay 2000).

We detected extended dust emission and resolved circumstellar material around the northern binary ( $\sim$ 0.'3 separation) of the quadruple system GG Tau (Leinert et al. 1993). Previous ${ }^{13} \mathrm{CO}$ line emission and $1.4-\mathrm{mm}$ continuum observations demonstrated that the circumbinary disk consists of two structural components: an 80-AU wide ring at a radius of 220 AU, and a fainter, more extended disk (Dutrey et al. 1994; Guilloteau et al. 1999). Given the complicated source geometry of GG Tau we have not attempted to deconvolve a simple Gaussian model from the 7-mm map.

The quadruple system UZ Tau could be resolved into the bright eastern and the (marginally detected) western components, both of which are known to harbour a binary system. Speckle and direct imaging observations resolved UZ Tau W into a binary system with a projected separation of $\sim 50 \mathrm{AU}$ (Ghez et al. 1993; Simon et al. 1995). Radial-velocity measurements showed UZ Tau E to be a spectroscopic binary with a projected semimajor axis of $\sim 0.1$ AU (Mathieu et al. 1996; Prato et al. 2002). We confirm the findings of Jensen et al. (1996), who noted a substantial reduction of millimetre emission around UZ Tau W compared to the tight binary UZ Tau E. In the former system, the two components have a separation comparable to a typical protoplanetary disk, leading to disk truncation and therefore smaller and less massive disks. The two components of UZ Tau E, on the other hand, apparently do not affect the common circumbinary disk in which they reside (Mathieu et al. 2000).

\subsection{Centimetre fluxes}

Centimetre emission could be detected for all five sources observed at $1.3,2.0$, and $3.6 \mathrm{~cm}$ (Table 3 ). The integrated fluxes of UZ Tau at 2.0 and $3.6 \mathrm{~cm}$ are below the $4-\sigma$ level. The counterjet of HL Tau mentioned in Sect. 3.1 can also be seen in the corresponding 1.3-cm image. Its position angle has been measured to $225^{\circ} \pm 20^{\circ}$, in agreement with the value measured from 

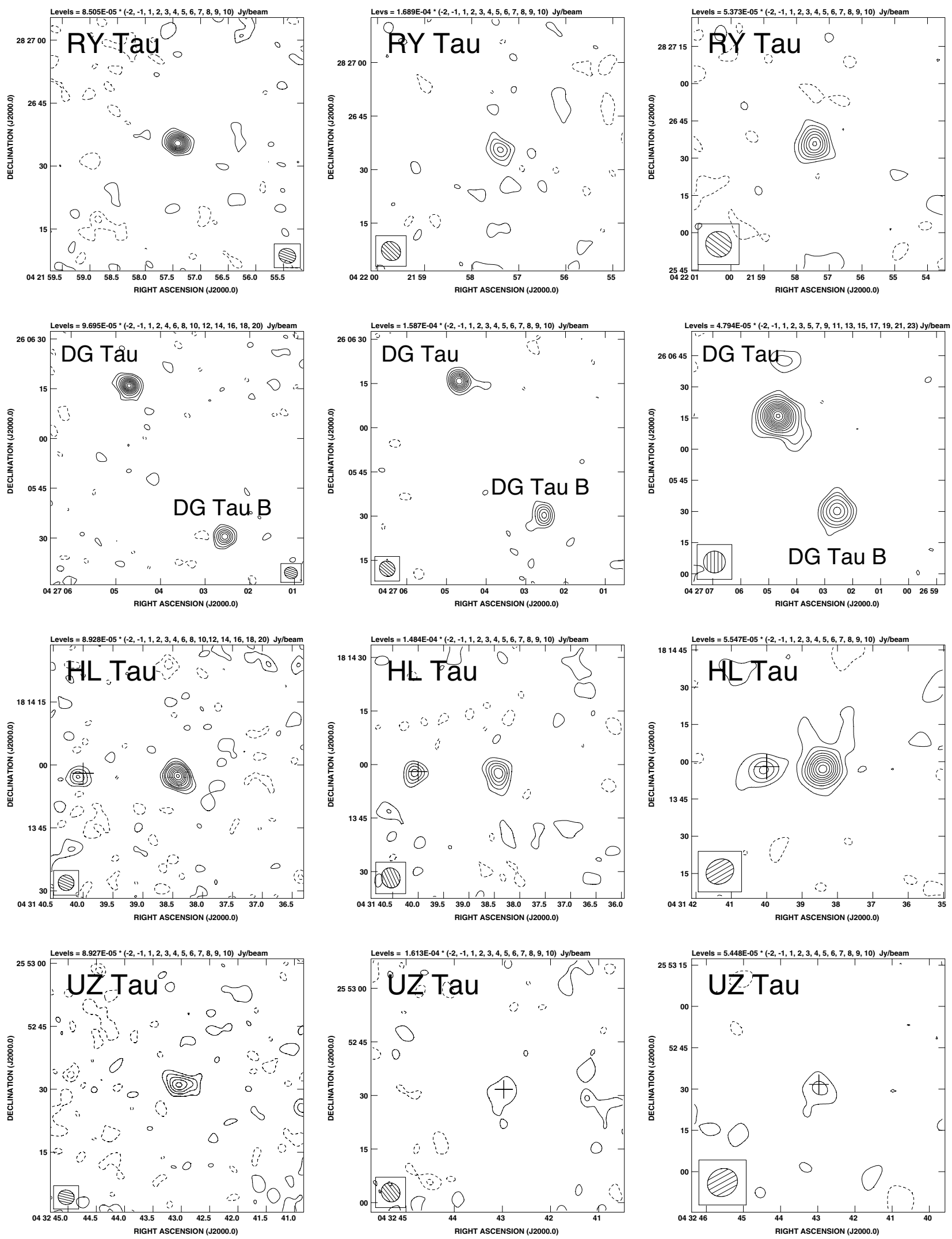

Fig. 2. VLA D-configuration images at $\lambda=1.3 \mathrm{~cm}$ (left column), $\lambda=2.0 \mathrm{~cm}$ (middle column), and $\lambda=3.6 \mathrm{~cm}$ (right column) for RY Tau, DG Tau and DG Tau B, HL Tau, and UZ Tau. Contour levels are given for each panel separately in multiples of $2 \sigma$ rms noise level. The average $1 \sigma \mathrm{rms}$ noise levels are approximately 50, 80, and $30 \mu \mathrm{Jy} / \mathrm{beam}$ at $1.3,2.0$, and $3.6 \mathrm{~cm}$, respectively. About $30^{\prime \prime}$ to the east of $\mathrm{HL}$ Tau lies XZ Tau (indicated by crosshairs). 
Table 2. Seeing-corrected 7-mm flux densities, spatial resolutions, and estimated source sizes. The stated uncertainties for the flux densities do not contain the uncertainty of the absolute flux calibration.

\begin{tabular}{|c|c|c|c|c|}
\hline Source & $\begin{array}{l}\text { Integrated flux } \\
(\mathrm{mJy})\end{array}$ & Disk resolved? & $\begin{array}{c}\text { Deconvolved source size } \\
(\mathrm{AU})\end{array}$ & $\begin{array}{l}\mathrm{PA} \\
\left({ }^{\circ}\right)\end{array}$ \\
\hline CY Tau & $1.19 \pm 0.26$ & no & - & - \\
\hline RY Tau & $2.97 \pm 0.29$ & yes & $180( \pm 30) \times 40( \pm 50)$ & $140 \pm 10$ \\
\hline FT Tau & $1.62 \pm 0.27$ & partially & $170( \pm 50) \times-$ & $60 \pm 20$ \\
\hline DG Tau B & $3.57 \pm 0.29$ & yes & $130( \pm 30) \times 40( \pm 50)$ & $50 \pm 30$ \\
\hline DG Tau & $5.30 \pm 0.37$ & partially & $110( \pm 30) \times-$ & $10 \pm 10$ \\
\hline HL Tau & $4.92 \pm 0.43$ & yes & $140( \pm 40) \times 80( \pm 40)$ & $170 \pm 30$ \\
\hline GG Tau & $3.24 \pm 0.42$ & - & & \\
\hline UZ Tau E & $1.84 \pm 0.28$ & partially & $120( \pm 50) \times-$ & $80 \pm 20$ \\
\hline DL Tau & $1.49 \pm 0.25$ & yes & $210( \pm 60) \times 140( \pm 100)$ & $110 \pm 40$ \\
\hline DM Tau & $0.74 \pm 0.20$ & - & & \\
\hline CI Tau & $0.76 \pm 0.17$ & partially & $220( \pm 60) \times-$ & $40 \pm 10$ \\
\hline DO Tau & $2.47 \pm 0.29$ & partially & $60( \pm 50) \times-$ & $70 \pm 30$ \\
\hline $\mathrm{LkCa} 15$ & $0.44 \pm 0.17$ & - & & \\
\hline GM Aur & $1.05 \pm 0.23$ & yes & $200( \pm 50) \times 60( \pm 70)$ & $10 \pm 20$ \\
\hline
\end{tabular}

the 7-mm map. The UZ Tau multiple system is not resolved into its eastern and western components.

Radio emission from low-mass pre-main-sequence stars is a common phenomenon (Güdel 2002). VLA observations of classical $\mathrm{T}$ Tauri stars at radio wavelengths revealed rising spectral indices and large angular sizes, interpreted as signs of wind emission (Cohen et al. 1982). Observational evidence and theoretical arguments favour focussed anisotropic outflows and collimated jets rather than uniform, isotropic mass flows from T Tauri stars (Cohen 1982; Cohen \& Bieging 1986). Wind emission is likely to contribute to the 7-mm continuum emission and has to be subtracted from the measured 7-mm flux densitites in order to accurately derive dust opacity indices from spectral slopes at millimetre wavelengths.

\subsection{Free-free emission}

At centimetre wavelengths the radio continuum of young stars is expected to be dominated by free-free radiation (bremsstrahlung) from ionized winds or outflows. There are, however, some observational indications that dust continuum emission may be a substantial source contributing to the centimetre fluxes of T Tauri stars (Rodriguez et al. 1994; Wilner et al. 1996, 2005).

With the exception of RY Tau, all other sources observed at $1.3 \mathrm{~cm}$ appear to be resolved, at least in one spatial direction. The position angles roughly match (within $20^{\circ}$ ) those derived from the 7-mm images. This finding supports the hypothesis that dust emission rather than an ionised wind is the main emission mechanism. For HL Tau the position angles differ by $\sim 40^{\circ}$, probably as a result of the bright counter-jet.

We measured the free-free spectrum for four sources by fitting a power law to the centimetre flux values (Fig. 3).
Table 3. Summary of centimetre observations. $1.3-\mathrm{cm}$ values corrected for seeing-related flux reduction. The uncertainty in the absolute flux density calibration is not included.

\begin{tabular}{lllc}
\hline \hline Wavelength & Source & $\begin{array}{l}\text { Integrated } \\
\text { flux }(\mathrm{mJy})\end{array}$ & $\begin{array}{c}\text { Detection } \\
(\sigma)\end{array}$ \\
\hline \multirow{2}{*}{$1.3 \mathrm{~cm}$} & RY Tau & $0.92 \pm 0.08$ & 12 \\
& DG Tau B & $1.23 \pm 0.09$ & 14 \\
& DG Tau & $2.17 \pm 0.11$ & 20 \\
& HL Tau & $1.63 \pm 0.11$ & 15 \\
& UZ Tau & $0.77 \pm 0.11$ & 7 \\
\hline \multirow{2}{*}{$.0 \mathrm{~cm}$} & RY Tau & $0.63 \pm 0.13$ & 5 \\
& DG Tau B & $0.80 \pm 0.13$ & 6 \\
& DG Tau & $1.33 \pm 0.15$ & 9 \\
& HL Tau & $0.88 \pm 0.14$ & 6 \\
& UZ Tau & $0.48 \pm 0.14$ & 3 \\
\hline \multirow{2}{*}{$3.6 \mathrm{~cm}$} & RY Tau & $0.31 \pm 0.04$ & 8 \\
& DG Tau B & $0.46 \pm 0.05$ & 9 \\
& DG Tau & $1.27 \pm 0.05$ & 25 \\
& HL Tau & $0.57 \pm 0.05$ & 11 \\
& UZ Tau & $0.10 \pm 0.03$ & 3 \\
\hline
\end{tabular}

The measured 2.0 and $3.6-\mathrm{cm}$ fluxes are assumed to originate from free-free radiation only. At higher frequencies, a reliable quantification of the corresponding contribution of thermal dust emission and free-free radiation is difficult; we assumed an equal mixture of dust and wind emission at $1.3 \mathrm{~cm}$. The spectral index $\alpha_{\mathrm{ff}}\left(S_{v} \propto v^{\alpha} \alpha_{\mathrm{ff}}\right)$ was found to be in the range -0.1 to +0.4 . A symmetric, ionized, and opaque wind with constant velocity has a spectral power index of 0.6 (Panagia \& Felli 1975; 

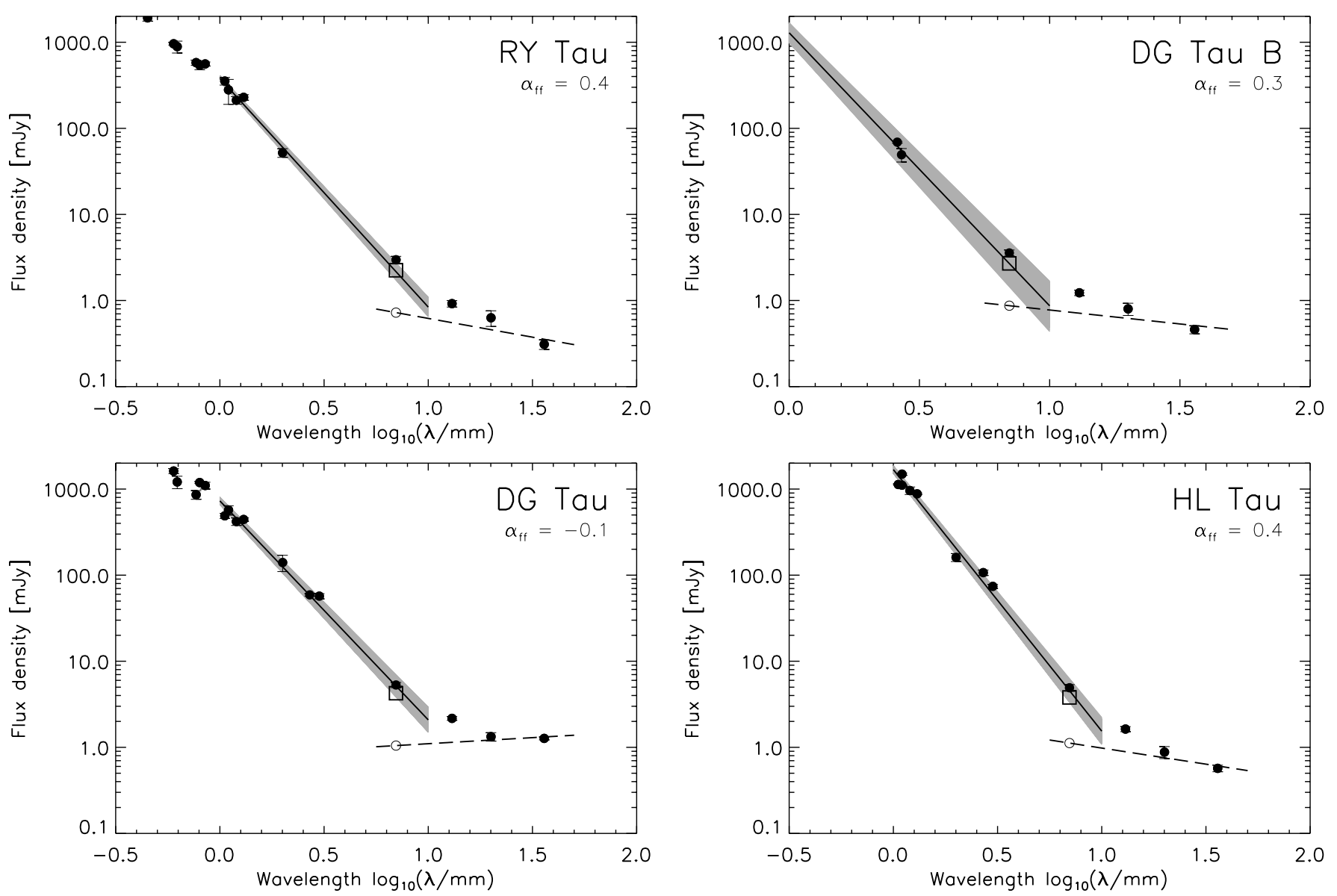

Fig. 3. Free-free emission contribution to $7-\mathrm{mm}$ flux densities. The dashed line represents the fit to the centimetre fluxes, assuming that $50 \%$ of the 1.3-cm emission arises from free-free radiation. The square depicts the corrected 7-mm flux after subtraction of the estimated free-free contribution (shown as open circle). The thick line shows the power-law fit to the millimetre data; the shaded region indicates the uncertainty range for the 1-7 mm slope. Data points shortwards of $\lambda=7 \mathrm{~mm}$ were compiled from the literature (Table 5).

Wright \& Barlow 1975; Olnon 1975). $\alpha_{\mathrm{ff}}=-0.1$ is expected for totally transparent (optically thin) free-free emission (Mezger et al. 1967).

For all four objects we extrapolated the free-free radio spectrum to $\lambda=7 \mathrm{~mm}$ to estimate the contribution of wind emission to the 7-mm fluxes. Subtracting the estimated free-free emission from the measured 7-mm value one obtains the thermal dust emission. We found that about $20 \%$ of the $7-\mathrm{mm}$ emission originates from free-free radiation; $80 \%$ are due to dust continuum emission. For sources where no centimetre data were available, we used these numbers to correct the 7-mm fluxes.

\subsection{Spectral indices and dust opacity indices}

The outer disk radii derived suggest that the emission is optically thin at $7 \mathrm{~mm}$. One can therefore directly use the slope of the millimetre spectral energy distribution $\left(F_{v} \propto v^{\alpha}\right)$ to derive a dust opacity law $\left(\kappa_{v} \propto v^{\beta}\right)$ (Beckwith \& Sargent 1991; Testi et al. 2003; Natta et al. 2004). Were the millimetre emission completely optically thin, i.e. without any contribution from the optically thick inner disk, one could simply read off the opacity index $\beta$ from the spectral index $\alpha$ via the relation $\beta=\alpha-2$.
There is, however, a non-negligible contribution from the optically thick part of the disk to the measured fluxes. Knowing this contribution will enable an improved estimate for the emissivity index $\beta$.

The revised relation between the opacity (emissivity) index $\beta$ and the observed spectral index $\alpha$ is given by $\beta \approx(\alpha-2) \times(1+\Delta)$, where $\Delta$ is the ratio of optically thick to optically thin disk emission given by $\Delta=-p \times\{(2-q) \ln [(1-p / 2) \bar{\tau}]\}^{-1}$ (Eq. (20) in Beckwith et al. 1990). Here $q, p$, and $\bar{\tau}$ are the power-law exponent of radial disk temperature, the power-law exponent of the surface density, and the average optical depth of the disk, respectively.

For the surface-density profile we adopted a value of $p=1.5$ throughout. The temperature profile index $q$ is uniquely determined by the slope of the spectral energy distribution in the optically thick regime, given by the relation $q=2 /\left(3-\alpha_{\mathrm{IR}}\right)$. We measured the spectral slope at far-infrared wavelengths (see Table 5), and derived temperature indices in the range of $q \approx 0.5-0.7$. Since $\Delta$ depends only weakly (logarithmically) on the average optical depth $\bar{\tau}$, an order-ofmagnitude estimate for this quantity will suffice for our purposes. $\bar{\tau}$ is a function of the opacity, mass, outer radius, and 
Table 4. Spectral slopes derived from power-law fitting in the range 1-7 mm using literature values (Table 5) and our 7-mm data (Col. 2). For objects without centimetre measurements, a $20 \%$ contribution of free-free emission was assumed (denoted by brackets in Col. 3). Spectral slopes corrected for free-free radiation are listed in Col. 4. The estimated dust opacity indices $\beta^{\sim}$ (Col. 5) were corrected for optically depth (using $\Delta=0.2$ ), yielding the final $\beta$ values (Col. 6).

\begin{tabular}{lccccr}
\hline \hline Source & $\begin{array}{c}\text { mm slope } \\
\alpha_{\mathrm{mm}}\end{array}$ & $\begin{array}{c}\text { f-f contribution } \\
\text { at } 7 \mathrm{~mm}(\%)\end{array}$ & $\begin{array}{c}\text { f-f corrected } \\
\alpha_{\text {corr }}\end{array}$ & $\begin{array}{c}\beta \text { estimate } \\
\beta^{\sim}=\alpha_{\text {corr }}-2\end{array}$ & $\begin{array}{r}\text { Opacity index } \\
\beta=(1+\Delta) \beta^{\sim}\end{array}$ \\
\hline RY Tau & $2.51 \pm 0.10$ & 24 & $2.66 \pm 0.09$ & $0.66 \pm 0.09$ & $0.8 \pm 0.1$ \\
FT Tau & $2.62 \pm 0.17$ & $(20)$ & $2.76 \pm 0.24$ & $0.76 \pm 0.24$ & $0.9 \pm 0.3$ \\
DG Tau B & $2.88 \pm 0.20$ & 24 & $3.17 \pm 0.19$ & $1.17 \pm 0.19$ & $1.4 \pm 0.2$ \\
DG Tau & $2.45 \pm 0.09$ & 20 & $2.54 \pm 0.11$ & $0.54 \pm 0.11$ & $0.7 \pm 0.1$ \\
HL Tau & $2.93 \pm 0.10$ & 23 & $3.04 \pm 0.12$ & $1.04 \pm 0.12$ & $1.3 \pm 0.1$ \\
UZ Tau E & $2.56 \pm 0.08$ & $(20)$ & $2.66 \pm 0.09$ & $0.66 \pm 0.09$ & $0.8 \pm 0.1$ \\
DL Tau & $2.73 \pm 0.11$ & $(20)$ & $2.82 \pm 0.14$ & $0.82 \pm 0.14$ & $1.0 \pm 0.2$ \\
CI Tau & $3.00 \pm 0.27$ & $(20)$ & $3.12 \pm 0.30$ & $1.12 \pm 0.30$ & $1.3 \pm 0.4$ \\
DO Tau & $2.29 \pm 0.06$ & $(20)$ & $2.38 \pm 0.07$ & $0.38 \pm 0.07$ & $0.5 \pm 0.1$ \\
GM Aur & $3.17 \pm 0.20$ & $(20)$ & $3.29 \pm 0.20$ & $1.29 \pm 0.20$ & $1.6 \pm 0.2$ \\
\hline
\end{tabular}

orientation of the disk (Eq. (16) in Beckwith et al. 1990). We estimate its approximate value by taking characteristic numbers for these parameters, i.e. disk masses from Beckwith et al. (1990) and disk radii from Table 2, for a circumstellar disk seen at random orientation. We found that $\bar{\tau}$ is of the order of $\sim 10^{-2}$. After inserting all parameters in the correction formula we derive $\Delta \approx 0.2$. The optical-depth correction slightly increases the opacity indices. The final $\beta$ values are given in Table 4 .

\section{Discussion}

One can use the millimetre spectral slope of circumstellar disks around pre-main-sequence stars to gain information on the characteristic dust grain size, provided the observed emission is largely optically thin. The magnitude of the dust opacity index indicates whether dust aggregates are small or large compared to the observing wavelength. The $\beta$ values are a robust measure of the characteristic grain size in the emitting region. At $7 \mathrm{~mm}$, we largely observe the thermal emission of cold dust in the outer parts of the disk midplane.

For dust grains with sizes of the order of a few tenths of a micron $\left(\ll \lambda_{\text {obs }} / 2 \pi\right)$, as present in the interstellar medium and in protostellar cores, the opacity index has been found to be $\beta \approx 2$ (Hildebrand 1983; Draine \& Lee 1984; Ossenkopf \& Henning 1994). For very large bodies $\left(\gg \lambda_{\text {obs }} / 2 \pi\right)$ that block radiation by virtue of their geometrical cross-section, the opacity is frequency-independent (grey opacity), $\beta=0$.

Particles of about the same size as the observing wavelength of $\lambda=7 \mathrm{~mm}$, i.e. pebble-sized particles, are expected to be in an intermediate regime (Beckwith et al. 2000). Strictly speaking, one has to include the refractive index $m=n+\mathrm{i} k$ of the absorbing material. At millimetre wavelengths, the optical constants for silicate grains are $n \approx 3$ and $k \simeq 0$, thus $|m| \approx 3$ (Laor \& Draine 1993; Henning \& Mutschke 1997; Mutschke et al. 1998). Thus opacity indices $\beta \lesssim 1$ suggest the presence of dust particles with sizes $\gtrsim \lambda / 2 \pi|m|$, i.e. with millimetre dimensions (and possibly even larger).

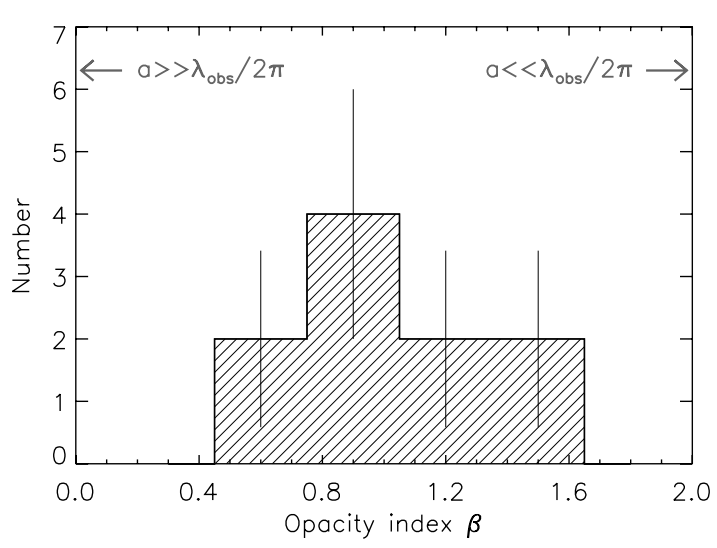

Fig. 4. Distribution of dust opacity indices. ISM-sized dust grains have $\beta \simeq 2$, while particles much larger than the observing wavelength $\lambda=7 \mathrm{~mm}$ have a frequency-independent opacity law $(\beta=0)$. The populated intermediate regime is indicative for the presence of millimetre-sized dust aggregates.

After correcting for free-free radiation and optically thick emission, we determined dust opacity indices $\beta$ in the range $0.5-1.6$ for 10 sources where the circumstellar disk could be spatially resolved (Fig. 4). Six objects have $\beta$ value $\lesssim 1$, a robust indication of agglomerated dust particles in the millimetre size regime. Four other sources have $\beta$ values between 1.3 and 1.6, lower than the dust opacity index $\beta \simeq 2$ of submicronsized particles as found in the interstellar medium. $\beta$ values smaller than 2 may also be explained by grain properties like shape, composition, conductivity, porosity, and crystallinity of the dust particles (Henning et al. 1995). Size, however, is the most important parameter influencing dust opacity indices. We deem unlikely the possibility that extreme dust compositions or particle structures cause the observed low $\beta$ values (Koerner et al. 1995; Draine 2005).

We underline the importance of proper free-free correction of the millimetre slopes, without which the opacity indices 


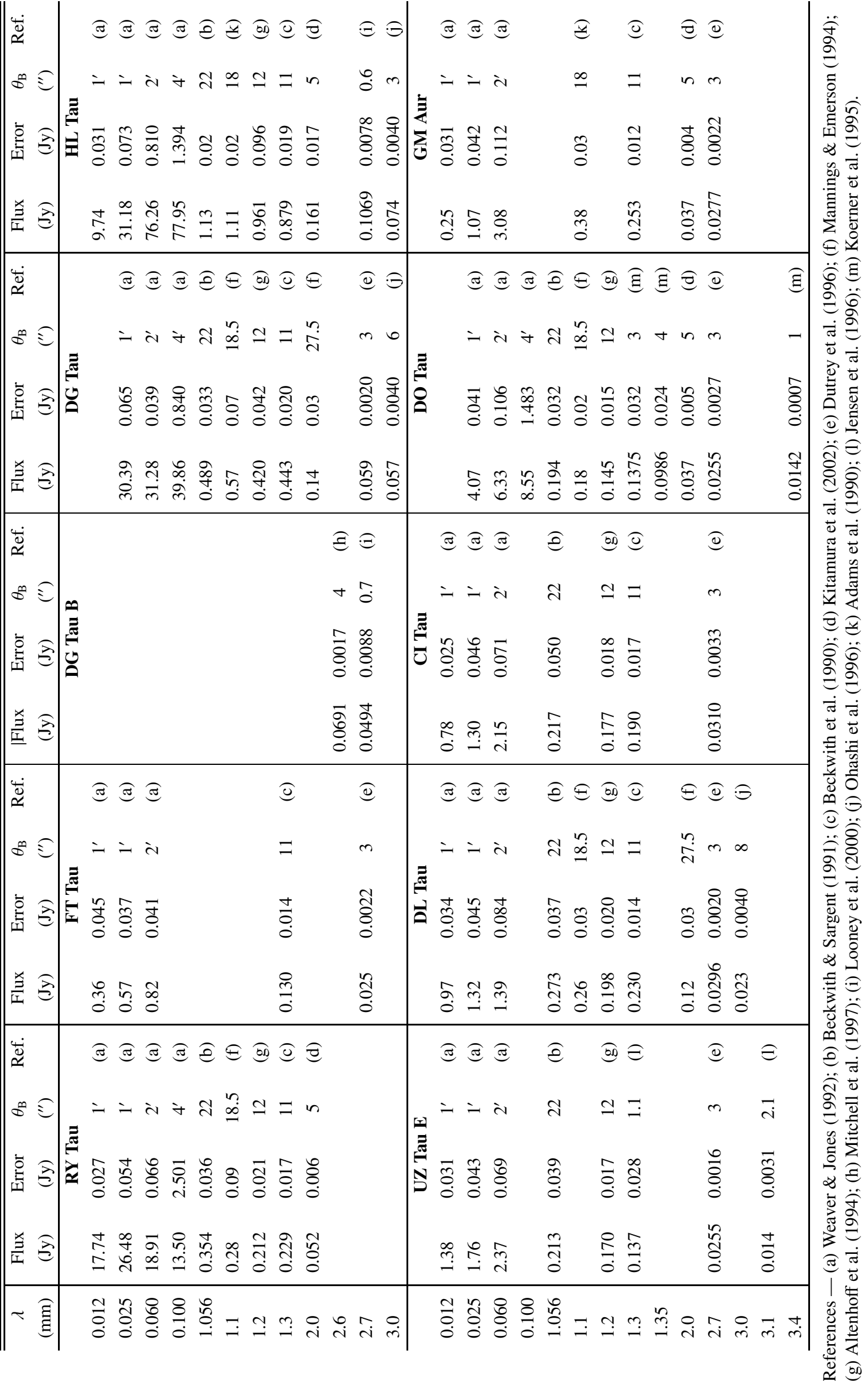


would be systematically smaller. The detection of emission at centrimetric wavelengths remains the only safe method to estimate the contamination of free-free radiation to the millimetre emission observed (Testi et al. 2001; Natta et al. 2004).

\section{Conclusion}

We presented continuum observations at $7 \mathrm{~mm}$ of a large sample of low-mass pre-main-sequence stars located in the TaurusAuriga star-forming region with ages between approximately $100 \mathrm{kyr}$ and 3 Myr. Circumstellar material could be spatially resolved for 10 sources, allowing us to derive the size of their circumstellar disk.

The deconvolved disk sizes (100-200 AU) effectively rule out the possibility that the $7 \mathrm{~mm}$ emission originates from small, optically thick disks containing submicron-sized dust particles. Knowing that the millimetre emission is mostly optically thin, we could directly translate the spectral indices $\alpha_{\mathrm{mm}}$ measured in the millimetre part of the spectral energy distribution into the frequency dependence of the dust opacity.

The power-law index $\beta$ is indicative for the characteristic size of the radiating dust particles. From our $7 \mathrm{~mm}$ observation and other millimetre fluxes compiled from the literature, we found $\beta$ values covering the range $0.5-1.6$. More than half of the objects where the disk surrounding the star could be spatially resolved show clear evidence for the presence of millimetre-sized dust particles, demonstrating the operation of dust grain growth processes in disks around T Tauri stars. The remaining sources may not require large dust aggregates and are consistent with submicron-sized dust as found in the interstellar medium.

Together with similar results for TW Hya, CQ Tau, and two Herbig Ae stars (HD 34282, HD 163296) (Calvet et al. 2002; Testi et al. 2003; Natta et al. 2004), a picture of grain growth to pebble-sized particles in disks around low- to intermediatemass pre-main-sequence stars is emerging. Further investigations of grain growth with existing and upcoming millimetre interferometers are needed to study where and how fast the building blocks of planetesimals are formed.

Millimetre observations trace the cold outer disk midplane where most of the disk mass is locked up. The grainsize indicator $\beta$ therefore only conveys information on that disk region. The surface layer of the inner disk is accessible through mid-infrared spectroscopy. The comparison of dustsize indicators from millimetre interferometry and mid-infrared spectroscopy, sampling different regions of circumstellar disks around $\mathrm{T}$ Tauri stars, may yield valuable insights into the physical processes that are thought to control the formation of planetesimals and the evolution of the circumstellar disk. Establishing the characteristic dust grain sizes at different locations in circumstellar disks holds the key to a better understanding of planet formation and disk evolution.

Acknowledgements. J.R. would like to thank Andreas Brunthaler (Joint Institute for VLBI in Europe, JIVE) and Hendrik Linz (MPIA, Heidelberg) for their helpful introduction into the world of $\mathcal{A I P S}$. We also thank Ralf Launhardt for a critical reading of the manuscript. Partial support for D.J.W. for this work was provided by NASA
Origins of Solar System Program Grant NAG5-11777.

This research has made extensive use of the SIMBAD database and VizieR catalogue service, operated at CDS, Strasbourg, France.

\section{References}

Adams, F. C., Lada, C. J., \& Shu, F. H. 1987, ApJ, 312, 788

Adams, F. C., Emerson, J. P., \& Fuller, G. A. 1990, ApJ, 357, 606

Altenhoff, W. J., Thum, C., \& Wendker, H. J. 1994, A\&A, 281, 161

Beckwith, S. V. W., \& Sargent, A. I. 1991, ApJ, 381, 250

Beckwith, S. V. W., Sargent, A. I., Chini, R. S., \& Güsten, R. 1990, AJ, 99, 924

Beckwith, S. V. W., Henning, T., \& Nakagawa, Y. 2000, Protostars and Planets IV, 533

Calvet, N., D'Alessio, P., Hartmann, L., et al. 2002, ApJ, 568, 1008

Carilli, C. L., Carlstrom, J. E., \& Holdaway, M. A. 1999, in Synthesis Imaging in Radio Astronomy II, ASP Conf. Ser. 180, 565

Carpenter, J. M., Wolf, S., Schreyer, K., Launhardt, R., \& Henning, T. 2005, AJ, 129, 1049

Cernicharo, J., Bachiller, R., \& Duvert, G. 1985, A\&A, 149, 273

Cohen, M. 1982, PASP, 94, 266

Cohen, M., \& Bieging, J. H. 1986, AJ, 92, 1396

Cohen, M., Bieging, J. H., \& Schwartz, P. R. 1982, ApJ, 253, 707

Draine, B. T. 2005, ApJ, submitted [arXiv: astro-ph/0507292]

Draine, B. T., \& Lee, H. M. 1984, ApJ, 285, 89

Dutrey, A., Guilloteau, S., \& Simon, M. 1994, A\&A, 286, 149

Dutrey, A., Guilloteau, S., Duvert, G., et al. 1996, A\&A, 309, 493

Duvert, G., Guilloteau, S., Ménard, F., Simon, M., \& Dutrey, A. 2000, A\&A, 355, 165

Elias, J. H. 1978, ApJ, 224, 857

Güdel, M. 2002, ARA\&A, 40, 217

Ghez, A. M., Neugebauer, G., \& Matthews, K. 1993, AJ, 106, 2005

Guilloteau, S., Dutrey, A., \& Simon, M. 1999, A\&A, 348, 570

Haisch, K. E., Lada, E. A., \& Lada, C. J. 2001, ApJ, 553, L153

Handa, T., Miyama, S. M., Yamashita, T., et al. 1995, ApJ, 449, 894

Hartmann, L. 2002, ApJ, 578, 914

Henning, T., Michel, B., \& Stognienko, R. 1995, Planet. Space Sci., 43, 1333

Henning, T., \& Mutschke, H. 1997, A\&A, 327, 743

Herbig, G. H., \& Bell, K. R. 1988, Lick Obs. Bull., 1111

Hildebrand, R. H. 1983, QJRAS, 24, 267

Jensen, E. L. N., Koerner, D. W., \& Mathieu, R. D. 1996, AJ, 111, 2431

Kenyon, S. J., \& Hartmann, L. 1995, ApJS, 101, 117

Kenyon, S. J., Dobrzycka, D., \& Hartmann, L. 1994, AJ, 108, 1872

Kitamura, Y., Momose, M., Yokogawa, S., et al. 2002, ApJ, 581, 357

Koerner, D. W., \& Sargent, A. I. 1995, AJ, 109, 2138

Koerner, D. W., Sargent, A. I., \& Beckwith, S. V. W. 1993, Icarus, 106, 2

Koerner, D. W., Chandler, C. J., \& Sargent, A. I. 1995, ApJ, 452, L69

Laor, A., \& Draine, B. T. 1993, ApJ, 402, 441

Leinert, C., Zinnecker, H., Weitzel, N., et al. 1993, A\&A, 278, 129

Loinard, L., Mioduszewski, A. J., Rodríguez, L. F., et al. 2005, ApJ, 619, L179

Looney, L. W., Mundy, L. G., \& Welch, W. J. 2000, ApJ, 529, 477

Mannings, V., \& Emerson, J. P. 1994, MNRAS, 267, 361

Mathieu, R. D., Martin, E. L., \& Magazzu, A. 1996, Bull. Amer. Astron. Soc., 28, 920

Mathieu, R. D., Ghez, A. M., Jensen, E. L. N., \& Simon, M. 2000, Protostars and Planets IV, 703

Mezger, P. G., Schraml, J., \& Terzian, Y. 1967, ApJ, 150, 807

Mitchell, G. F., Sargent, A. I., \& Mannings, V. 1997, ApJ, 483, L127 
Mundt, R., Bührke, T., \& Ray, T. P. 1988, ApJ, 333, L69

Mutschke, H., Begemann, B., Dorschner, J., et al. 1998, A\&A, 333, 188

Najita, J., Carr, J. S., \& Mathieu, R. D. 2003, ApJ, 589, 931

Natta, A., Testi, L., Neri, R., Shepherd, D. S., \& Wilner, D. J. 2004, A\&A, 416, 179

Ohashi, N., Hayashi, M., Kawabe, R., \& Ishiguro, M. 1996, ApJ, 466, 317

Olnon, F. M. 1975, A\&A, 39, 217

Ossenkopf, V., \& Henning, T. 1994, A\&A, 291, 943

Panagia, N., \& Felli, M. 1975, A\&A, 39, 1

Prato, L., Simon, M., Mazeh, T., Zucker, S., \& McLean, I. S. 2002, ApJ, 579, L99

Rodriguez, L. F., Canto, J., Torrelles, J. M., et al. 1994, ApJ, 427, L103

Simon, M., Ghez, A. M., Leinert, C., et al. 1995, ApJ, 443, 625
Strom, K. M., Strom, S. E., Edwards, S., Cabrit, S., \& Skrutskie, M. F. 1989, AJ, 97, 1451

Testi, L., Natta, A., Shepherd, D. S., \& Wilner, D. J. 2001, ApJ, 554, 1087

Testi, L., Natta, A., Shepherd, D. S., \& Wilner, D. J. 2003, A\&A, 403, 323

Weaver, W. B., \& Jones, G. 1992, ApJS, 78, 239

Wilner, D. J. 1998, unpublished data

Wilner, D. J., D'Alessio, P., Calvet, N., Claussen, M. J., \& Hartmann, L. 2005, ApJ, 626, L109

Wilner, D. J., Ho, P. T. P., Kastner, J. H., \& Rodríguez, L. F. 2000, ApJ, 534, L101

Wilner, D. J., Ho, P. T. P., \& Rodriguez, L. F. 1996, ApJ, 470, L117

Wilner, D. J., \& Lay, O. P. 2000, Protostars and Planets IV, 509

Wright, A. E., \& Barlow, M. J. 1975, MNRAS, 170, 41 